\title{
Brightness Compensation Investigation for Power-Efficient System
}

\author{
Gwanggil Jeon \\ Department of Embedded Systems Engineering, Incheon National University \\ 119 Academy-ro, Yeonsu-gu, Incheon 406-772, Korea \\ gjeon@incheon.ac.kr
}

Abstract

This paper presents brightness compensation and enhancement method for powerconstrained condition. The proposed method is useful for low-power device We assumed two conditions: half and quarter power-consumption. We compare two brightness enhancement methods in both scenarios, histogram equalization and contextual and variational contrast enhancement methods. Both methods are contras adjustment approgches using the given images histogram. Simulation results thoroughly repart objective and subjective performance.

Keywords: brightness compensation, power-efficient system, histogram equalization

\section{Introduction}

Nowadays, various displays are adopted in various devices, such as mobile TV or phones, portable media players, personal digital assistants, and notebooks [1]. The purpose of all devices is delivering information, however themain challenge and limitation of their usage is battery capacity [2]. Especially, watching multimedia such as pictures or videos consumes more battery than other activity. Although many researchers have been studying display techniques, the low power consumption is still important and unsolved issue yet.

Contrast enhancement often generates impractical effects in pictures [3]. However, contrast enhancement is somehow effective for scientific scene such as thermal or satellite images. Meanwhile, contrast enhancement can generate undesirable effects when it is applied to low color depth images [4]. In this paper we assume two scenarios, half- and quarter power power-consumption.

To show high color fidelity image with less energy consumption, some researchers propose various ideas [5]. For example, sender sends half contrast image and receiver can reproduce full contrast înage. However, half contrast image looks very dark especially in daylight and may harm hurnan visual system [6]. Therefore contrast enhancement method is important to be studied.

Aften grayscale contrast enhancement is performed, this can be applied to color image case. One can apply the same approach in each color components, red, green, and blue. However, implementing the identical approach on the red, green, and blue channels of color image can produce drastic change of image. To alleviate this issue, YUV transform can be firstly applied. This can prevent white balance error. To reduce energy consumption by the backlight, two enhancement methods were assumed in this paper. One is histogram equalization and the other one is contextual and variational contrast (CVC) enhancement.

This paper is organized as follows. Section 2 explains details of both contrast enhancement methods. Section 3 shows the simulation results with four metrics. Conclusion remarks are shown in Section 4. 


\section{Histogram Equalization and Contextual and Variational Contrast Enhancement}

There have been various contrast enhancement histogram equalization methods which were presented by various authors with different purpose and complexity. The contrast enhancement is widely used for low contract image. Especially, histogram equalization method is one of broadly used techniques to enlarge intensities of low-contrast image. By applying contrast enhancement, the number of pixels of each intensity becomes similar to each other. The CVC enhancement method is another method which is recently introduced. In this paper, we use two methods to design and investigate a power efficient contrast enhancement system. Here are reviews of both methods. Details of histogram equalization and CVC can be found [7].

Figure 1 shows the flowchart of the proposed method.

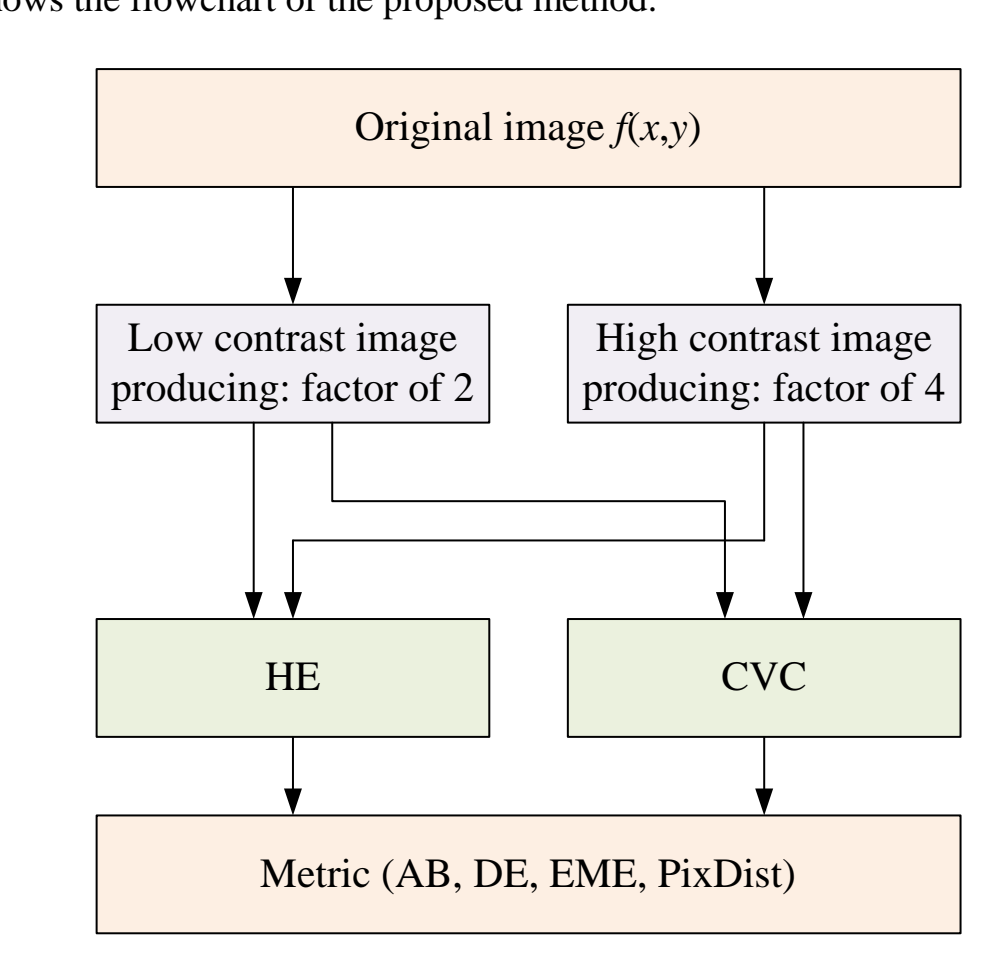

\section{Figure 1. Flowchart of the Proposed Method}

For gray cale image, the probability of level $i$ image is,

$$
p(i)=\frac{n_{i}}{n},
$$

where $i$ is less than $L$ and bigger than $0 . L$ is normally determined as 256 as we assume gray scale image. The cumulative distribution function, $\mathrm{CDF}$, is

$$
C D F(i)=\sum_{j=0}^{i} p(j)
$$

When $i$ is $L-1$, CDF is

$$
C D F(L-1)=1 .
$$

The output histogram $h_{\mathrm{HE}}$ is defined as Eq. (4).

$$
h_{H E}=\frac{L-1}{1^{T} h_{\text {ori }}} h_{\text {ori }} .
$$


where $h_{\text {ori }}$ are original histogram. The details of $h_{\mathrm{CVC}}$ are modified version of histogram equalization which can be found [7].

Figure 2 shows the code of histogram equalization.

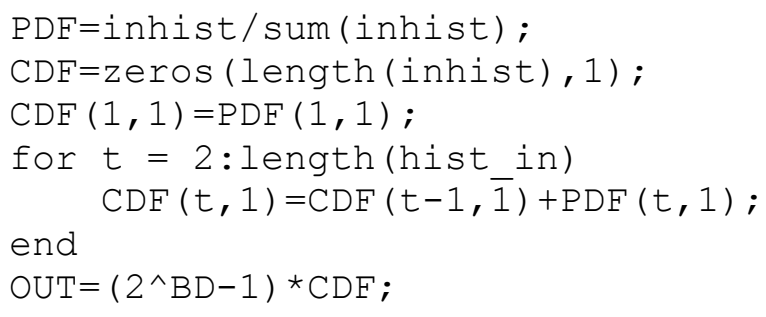

Figure 2. Histogram Equalization Software

Here, parameter BD stands for bit depth, which is 8 for gray scale inage, Figure 3 shows the step of contextual and variational contrast.

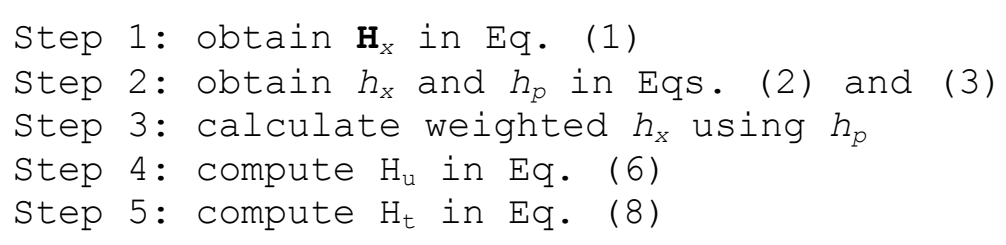

\section{Figure 3. Contextual and Variational Contrast Steps}

The contrast enhancement method is originally generated for gray scale image. However, as most images are color, we dan apply contrast enhancement methods to color image. We assume the obtained rêsults ean be applied to color images. To do so, one can convert RGB image into YUV and apply the methold in Y channel only, and preserve chrominance channels, $\mathrm{U}$ and V. Figure 4 shows the illustration of HE and CVC on \#22 to \#25 Zahra imageset. Line in red shows HE method results, and blue line shows CVC method result.

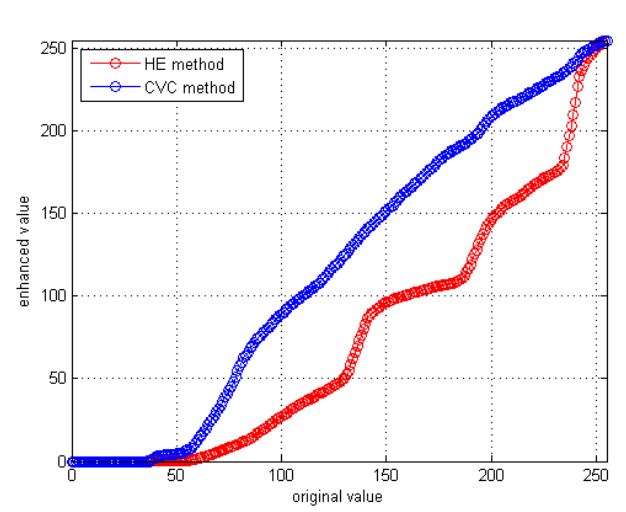

(a)

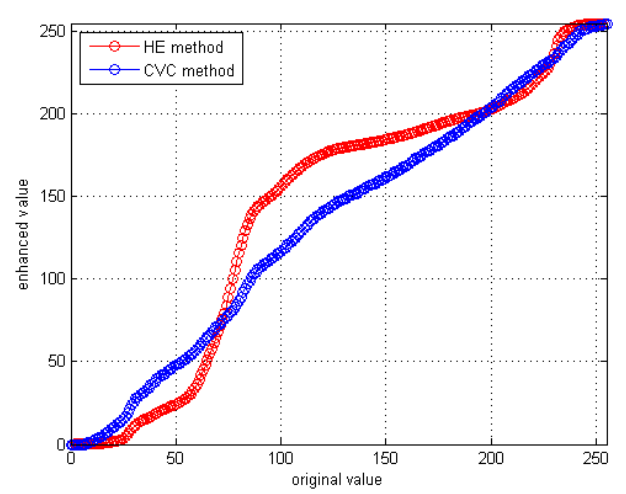

(b) 


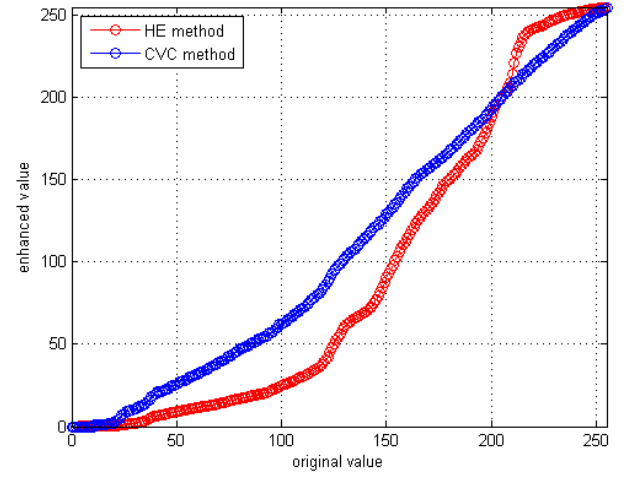

(c)

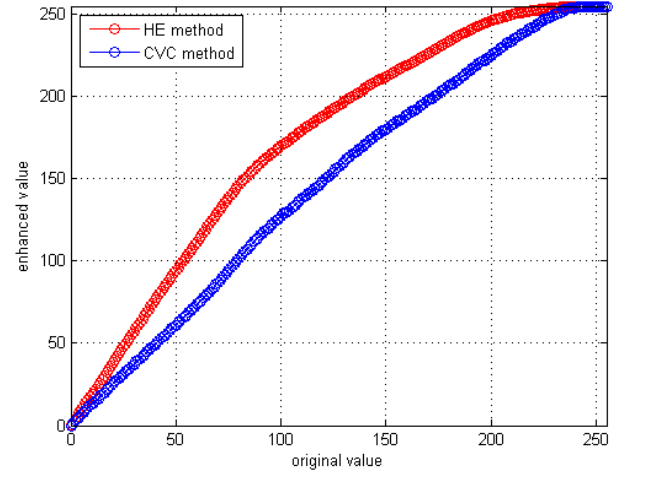

(d)

Figure 4. Illustration of HE and CVC for (a) \#22, (b) \#23, (c) \#24, and (d) \#25 Zahra imageset; Line in Red Shows HE Method Result and Line in Blue Shows CVC Method Result

\section{Experimental Results}

We use standard test images from Zahra dataset to evaluate an compare HE and CVC under two conditions, half power-consumption and quarter power-consumption. The parameters for CVC is $w \times w=7 \times 7$ adjacent block and $\alpha \beta$, and $\gamma$ are 0.33 , respectively.

To evaluate subjective performance, we used Courimages, \#2, \#3, \#13, and \#22. It is hard to evaluate contrast enhancementas it is hard to quantify an improved perception of an image. In general, PSNR or MSE metrics are widely used, however they have original images which are known as ground truth fowever, in our case, there is no ground truth to catch up with. Therefore, we used four metrics, absolute mean brightness error (AB) [8], discrete entropy (DE) [9], measure of enhancement [10], and pixel distance.

Figure 5 shows original four mages and Figures 6 and 7 show half and quarter powered original images respectively 2

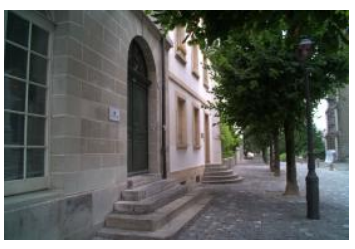

(a)

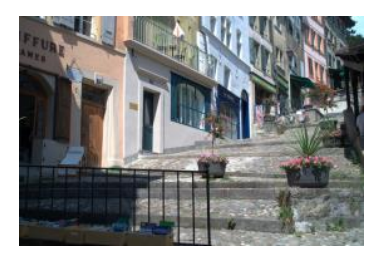

(b)

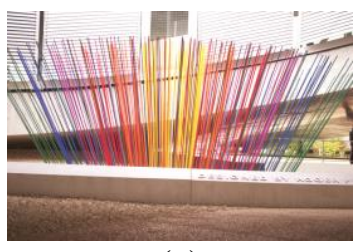

(c)

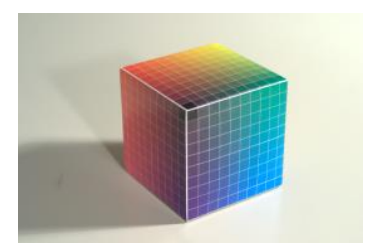

(d)

Figure 5. Original Zahra Image: (a) \#2, (b) \#3, (c) \#13, and (d) \#22

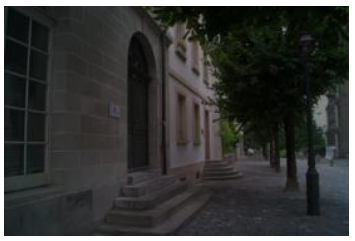

(a)

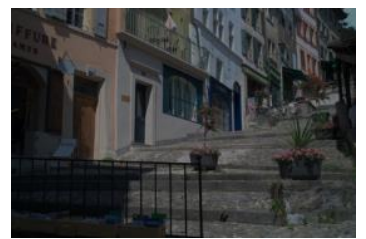

(b)

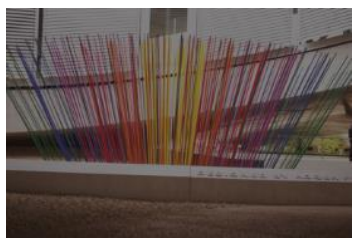

(c)

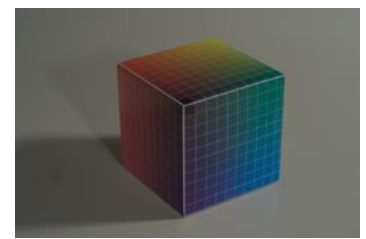

(d)

Figure 6. Original Zahra Images with Half Power-consumption: (a) \#2, (b) \#3, (c) \#13, and (d) \#22 


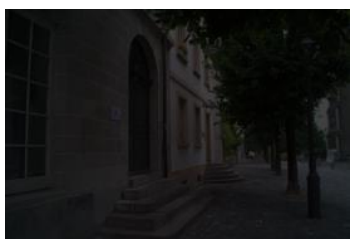

(a)

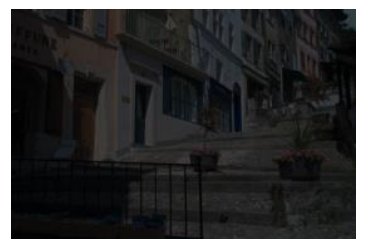

(b)

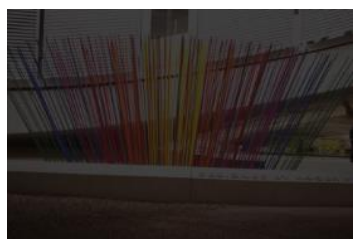

(c)

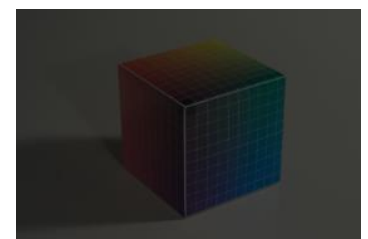

(d)

Figure 7. Original Zahra Images with Quarter Power-consumption: (a) \#2, (b) \#3, (c) \#13, and (d) \#22

Figures 8-11 show subjective performance comparison for half power-consumed on four Zahra image. And Figures 12-15 show subjective performance comparison for quarter powerconsumed on four Zahra image.

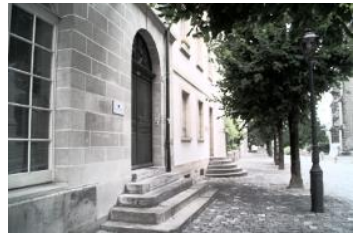

(a)

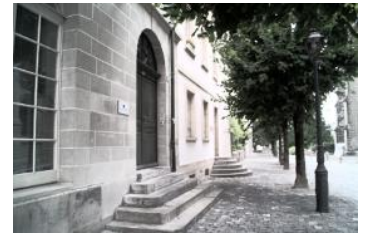

(b)

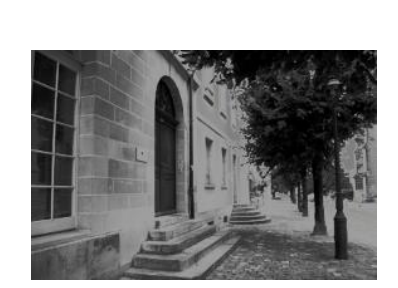

(c)

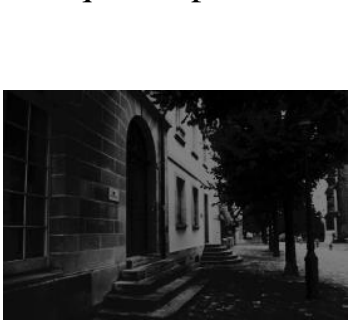

(d)

Figure 8. Subjective Performance Comparison for Half Power-consumed \#2 Zahra image: (a) HE result, (b) CVC result, (c) difference between HE and original, and (d) Difference between CVC and Original

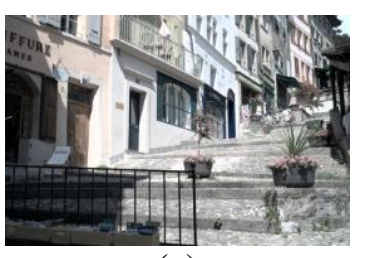

(a)

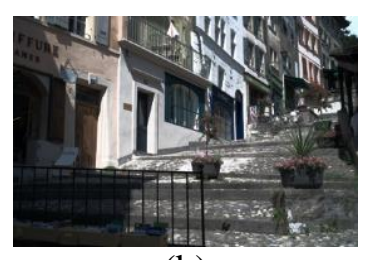

(b)

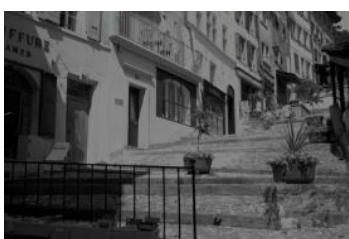

(c)

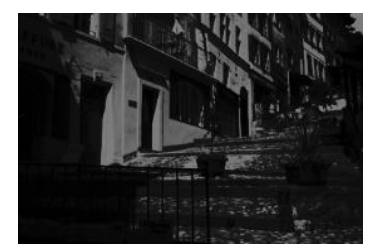

(d)

Figure 9. Subjective Performance Comparison for Half Power-consumed \#3 Zahra Image: (a) HE result, (b) CVC result, (c) difference between HE and

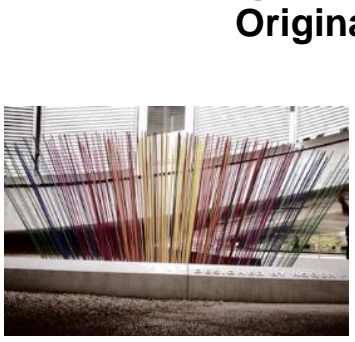

(a)

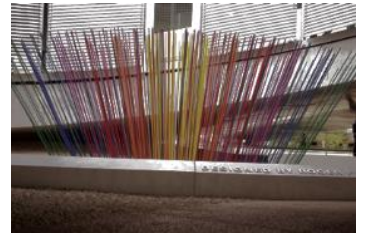

(b)

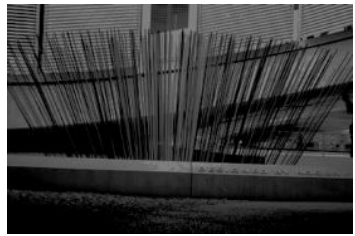

(c)

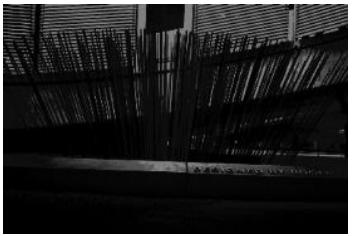

(d)

Figure 10. Subjective Performance Comparison for Half Power-consumed \#13 Zahra Image: (a) HE result, (b) CVC result, (c) difference between HE and original, and (d) difference between CVC and original 


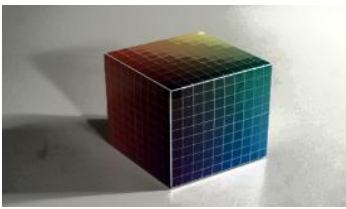

(a)

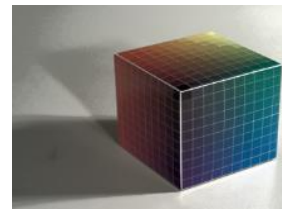

(b)

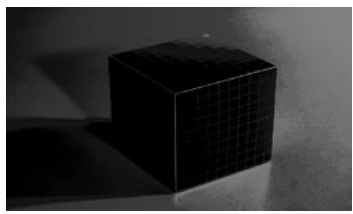

(c)

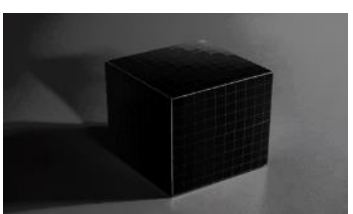

(d)

Figure 11. Subjective Performance Comparison for Half Power-consumed \#22 Zahra Image: (a) HE Result, (b) CVC Result, (c) Difference between HE and Original, and (d) Difference between CVC and Original

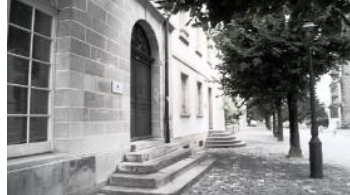

(a)

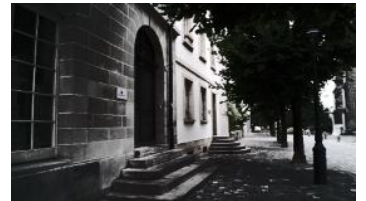

(b)

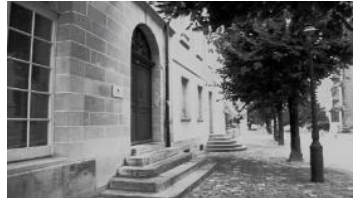

(c)

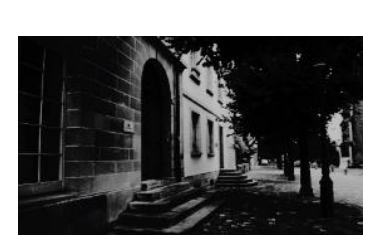

(d)

Figure 12. Subjective Performance Comparison for Quarter Power-consumed \#2 Zahra Image: (a) HE Result, (b) CVC Result, (c) Difference between HE and Original, and (d) Difference between $\mathrm{CVC}$ and Original

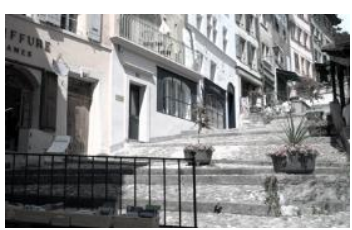

(a)

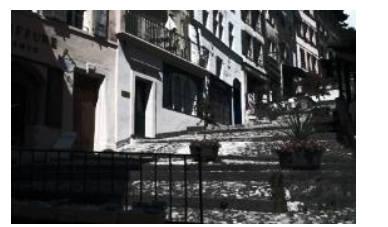

(b)

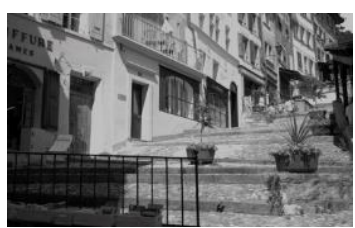

(c)

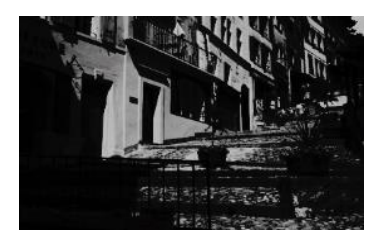

(d)

Figure 13. Subjective Performance Comparison for Quarter Power-consumed \#3 Zahra Image:(a) HE Result, (b) CVC Result, (c) Difference between HE and Original, and (d) Difference between CVC and Original

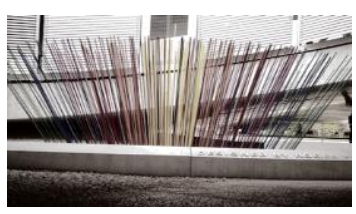

(a)

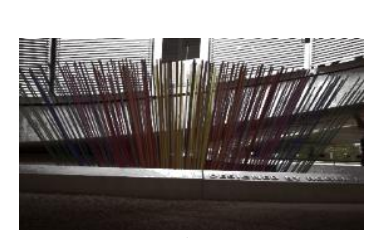

(b)

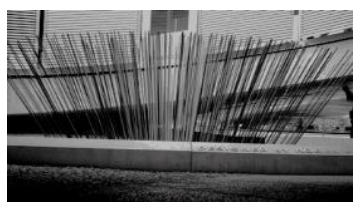

(c)

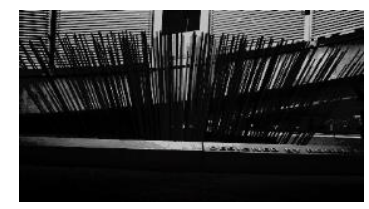

(d)

Figure 14. Subjective Performance Comparison for Quarter Power-consumed \#13 Zahra Image: (a) HE Result, (b) CVC Result, (c) Difference between HE and Original, and (d) Difference between CVC and Original

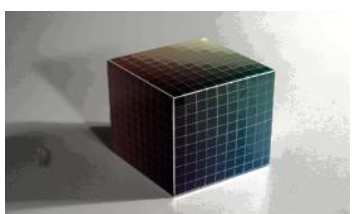

(a)

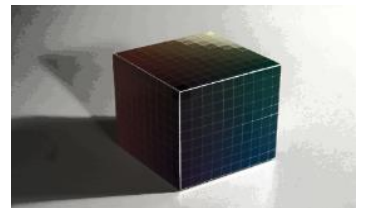

(b)

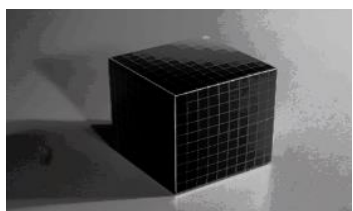

(c)

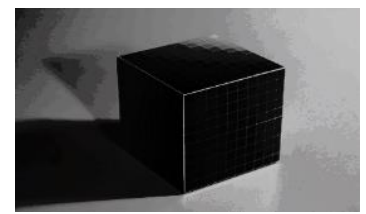

(d)

Figure 15. Subjective Performance Comparison for Quarter Power-consumed \#22 Zahra Image: (a) HE Result, (b) CVC Result, (c) Difference between HE and Original, and (d) Difference between CVC and original 
To assess objective performance, we used 25 images of Zahra imageset. Four metrics were used, and they are absolute mean brightness error (AB), discrete entropy (DE), measure of enhancement, and pixel distance. Table 1 and Figure 16 show four metrics' performance results on $\mathrm{HE}$ and $\mathrm{CVC}$ methods for half power consumption case.

Table 1. Objective Performance Results between Two Methods on Four Metrics (Half Power-consumption)

\begin{tabular}{|c|c|c|c|c|c|c|c|c|}
\hline Metric & \multicolumn{2}{|c|}{$\mathrm{AB}$} & \multicolumn{2}{|c|}{ DE } & \multicolumn{2}{|c|}{ EME } & \multicolumn{2}{|c|}{ PixDist } \\
\hline Method & $\mathrm{HE}$ & CVC & $\mathrm{HE}$ & CVC & $\mathrm{HE}$ & CVC & $\mathrm{HE}$ & CVC \\
\hline 1 & 128.67 & 85.57 & 6.89 & 6.88 & 30.35 & 29.65 & 42.42 & 32.15 \\
\hline 2 & 129.19 & 72.85 & 6.33 & 6.40 & 25.27 & 25.14 & 42.43 & 29.51 \\
\hline 3 & 128.64 & 84.07 & 6.79 & 6.84 & 26.03 & 26.86 & 42.41 & 33.96 \\
\hline 4 & 130.55 & 99.56 & 5.97 & 5.99 & 21.96 & 18.28 & 43.06 & 33.42 \\
\hline 5 & 130.01 & 62.94 & 5.93 & 6.03 & 41.901 & 35.53 & 42.29 & 26.78 \\
\hline 6 & 129.58 & 120.46 & 6.19 & 6.22 & 2402 & 15.35 & 42.62 & 40.04 \\
\hline 7 & 129.59 & 68.18 & 6.19 & 6.28 & 34.27 & 27.52 & 42.20 & 26.93 \\
\hline 8 & 129.73 & 93.35 & 6.01 & 6.09 & 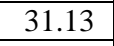 & 1780 & 42.33 & 27.38 \\
\hline 9 & 129.14 & 87.51 & 6.35 & 6.42 & 28.77 & 2249 & 42.44 & 32.99 \\
\hline 10 & 129.11 & 107.73 & 6.48 & 6.50 & $36.40^{\prime}$ & 25.19 & 42.40 & 40.13 \\
\hline 11 & 129.40 & 76.44 & 6.34 & 60.40 & 35.67 & 26.73 & 42.09 & 31.84 \\
\hline 12 & 129.04 & 102.61 & 6.52 & 6.55 & 29.34 & 21.32 & 42.20 & 40.79 \\
\hline 13 & 129.39 & 101.57 & 6.46 & 6.41 & 35.71 & 25.13 & 42.97 & 33.15 \\
\hline 14 & 133.16 & 123.53 & 6.03 & 6.08 & 29.39 & 24.32 & 45.85 & 46.60 \\
\hline 15 & 129.57 & 109.94 & 629 & 632 & 25.22 & 19.07 & 43.00 & 37.34 \\
\hline 16 & 129.21 & 117.85 & (6.4) & 6.43 & 26.29 & 15.77 & 42.70 & 30.23 \\
\hline 17 & 128.77 & 100.61 & 6.76 & 6.79 & 23.12 & 21.69 & 42.37 & 45.42 \\
\hline 18 & 129.11 & 147.90 & 6.46 & (6) 45 & 18.08 & 11.43 & 42.59 & 47.34 \\
\hline 19 & 129.25 & 81.50 & 6.38 & 6.42 & 34.70 & 26.40 & 42.21 & 34.81 \\
\hline 20 & 129.45 & - 107.15 & $6.3 \mathrm{~K}$ & 6.32 & 13.51 & 11.76 & 42.95 & 38.45 \\
\hline 21 & 130.42 & 153,28 & 5.85 & 5.86 & 21.35 & 11.13 & 43.40 & 42.61 \\
\hline 22 & 130.25 & 143.42 & 603 & 6.04 & 11.58 & 6.29 & 43.20 & 41.14 \\
\hline 23 & 129.87 & 90.02 & 626 & 6.18 & 24.90 & 17.87 & 42.24 & 36.37 \\
\hline 24 & 129.58 & 99.30 & 6.22 & 6.30 & 24.16 & 13.95 & 42.86 & 23.71 \\
\hline 25 & 128.88 & 7286 & 6.62 & 6.65 & 27.45 & 35.09 & 42.22 & 34.12 \\
\hline Avg. & 129.58 & $100.4 \mathrm{Y}$ & 6.32 & 6.36 & 27.22 & 21.27 & 42.70 & 35.49 \\
\hline
\end{tabular}

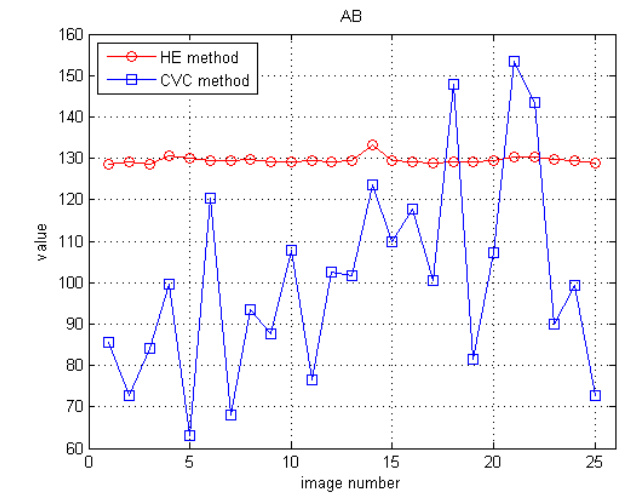

(a)

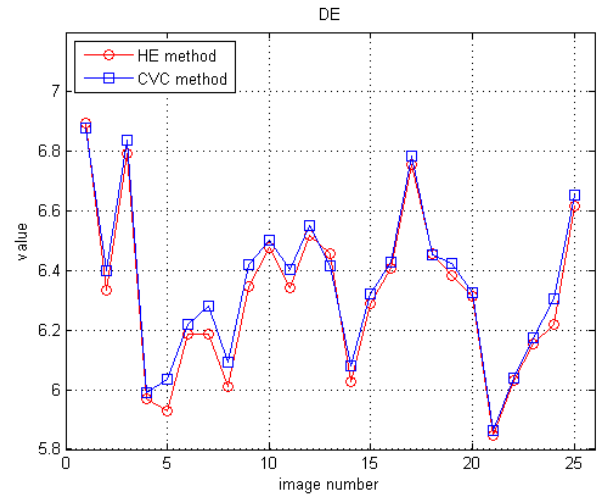

(b) 


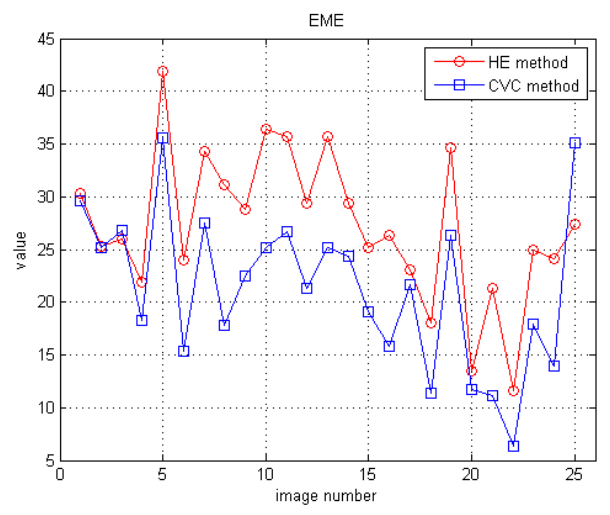

(c)

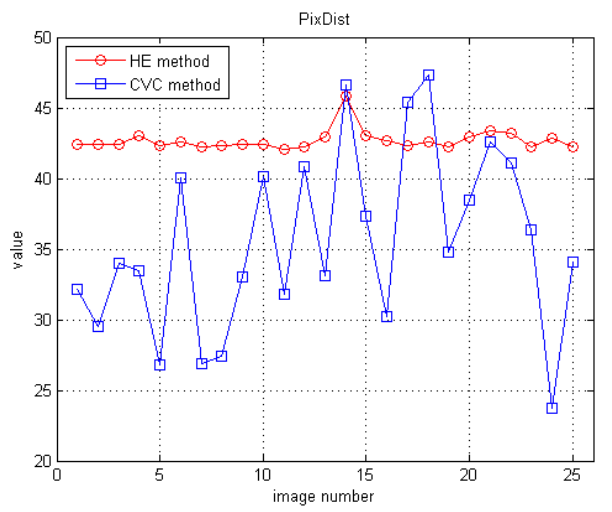

(d)

Figure 16. Objective Performance Results between Two Methods (Half PowerConsumption): (a) AB, (b) DE, (c) EME, and (d) PixDist

Table 2 and Figure 17 show four metrics' performance results on HE and CVC methods for quarter power consumption case.

Table 2. Objective Performance Results between Two Methods on Four Metrics (Quarter Power-consumption)

\begin{tabular}{|c|c|c|c|c|c|c|c|c|}
\hline Metric & \multicolumn{2}{|c|}{$\mathrm{AB}$} & $8<$ & & \multicolumn{2}{|c|}{ EME } & \multicolumn{2}{|c|}{ PixDist } \\
\hline Method & $\mathrm{HE}$ & CVC & $\mathrm{HE}$ & $\mathrm{CVC}^{\prime}$ & $\mathrm{HE}$ & CVC & $\mathrm{HE}$ & $\mathrm{CVC}$ \\
\hline 1 & 129.69 & 64.62 & 5.90 & 5.90 & 29.83 & 30.86 & 42.32 & 31.89 \\
\hline 2 & 130.96 & 57.57 & 5.40 & A 5.43 & 24.69 & 32.08 & 42.30 & 30.63 \\
\hline 3 & 129.81 & 66.76 & 5.83 & 5.83 & 25.68 & 33.78 & 42.31 & 34.85 \\
\hline 4 & 133.63 & 89.04 & 5.08 & 4.99 & 18.24 & 23.77 & 43.56 & 39.13 \\
\hline 5 & 132.37 & $50 . \overline{1}$ & 5.01 & 5.04 & 39.05 & 44.61 & 42.02 & 27.79 \\
\hline 6 & 131.80 & 108.87 & 5.23 & 5.26 & 20.58 & 17.03 & 42.79 & 45.09 \\
\hline 7 & 131.54 & 51.54 & 5.26 & 5.30 & 33.50 & 31.60 & 41.91 & 26.97 \\
\hline 8 & 131.85 & 73.59 & 5.07 & 5.11 & 30.72 & 22.99 & 42.13 & 32.38 \\
\hline 9 & 130.85 & 74.16 & 5.39 & 5.42 & 26.11 & 27.03 & 42.38 & 36.43 \\
\hline 10 & 130.65 & 90.72 & 5.51 & 5.52 & 36.39 & 27.07 & 42.27 & 43.84 \\
\hline 11 & 131.51 & 64.09 & 5.40 & 5.41 & 35.75 & 30.68 & 41.61 & 32.89 \\
\hline 12 & 130.49 & 91.56 & 5.55 & 5.56 & 27.81 & 23.65 & 41.92 & 44.53 \\
\hline 13 & 130.76 & 77.72 & 5.53 & 5.49 & 33.88 & 28.54 & 42.91 & 34.79 \\
\hline 14 & 134.39 & 106.65 & 5.24 & 5.26 & 28.48 & 29.16 & 45.88 & 50.91 \\
\hline 15 & 131.69 & 94.47 & 5.36 & 5.32 & 24.84 & 24.79 & 43.43 & 42.33 \\
\hline 16 & 130.91 & 98.32 & 5.43 & 5.43 & 23.65 & 16.03 & 42.87 & 37.80 \\
\hline 17 & 130.01 & 87.79 & 5.80 & 5.80 & 18.89 & 25.94 & 42.17 & 47.10 \\
\hline 18 & 130.82 & 135.04 & 5.50 & 5.51 & 16.55 & 12.11 & 42.66 & 50.96 \\
\hline 19 & 130.88 & 67.35 & 5.44 & 5.45 & 35.19 & 30.87 & 41.93 & 36.78 \\
\hline 20 & 131.46 & 94.61 & 5.33 & 5.32 & 12.88 & 15.75 & 43.35 & 43.62 \\
\hline 21 & 133.13 & 139.55 & 4.89 & 4.90 & 19.20 & 12.50 & 44.09 & 48.30 \\
\hline 22 & 132.80 & 126.27 & 5.06 & 5.04 & 11.24 & 7.36 & 43.75 & 47.50 \\
\hline 23 & 132.16 & 73.27 & 5.24 & 5.16 & 23.90 & 21.43 & 41.90 & 38.17 \\
\hline 24 & 131.69 & 75.23 & 5.33 & 5.31 & 24.14 & 16.97 & 43.10 & 28.77 \\
\hline 25 & 130.27 & 59.62 & 5.64 & 5.66 & 25.99 & 44.39 & 41.90 & 34.30 \\
\hline Avg. & 131.44 & 84.62 & 5.37 & 5.38 & 25.89 & 25.24 & 42.70 & 38.71 \\
\hline
\end{tabular}




\section{Conclusions}

In this paper, we investigated contrast enhancement methods' performance. Subjective and objective performance results are shown in simulation results section. The proposed brightness compensation method works well for half and quarter power-consumed devices.

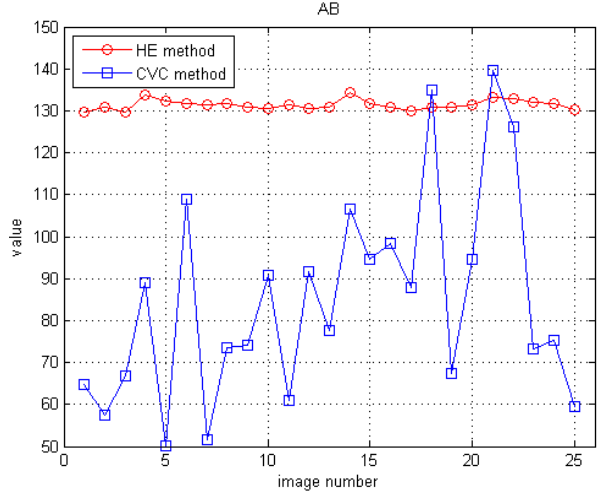

(a)

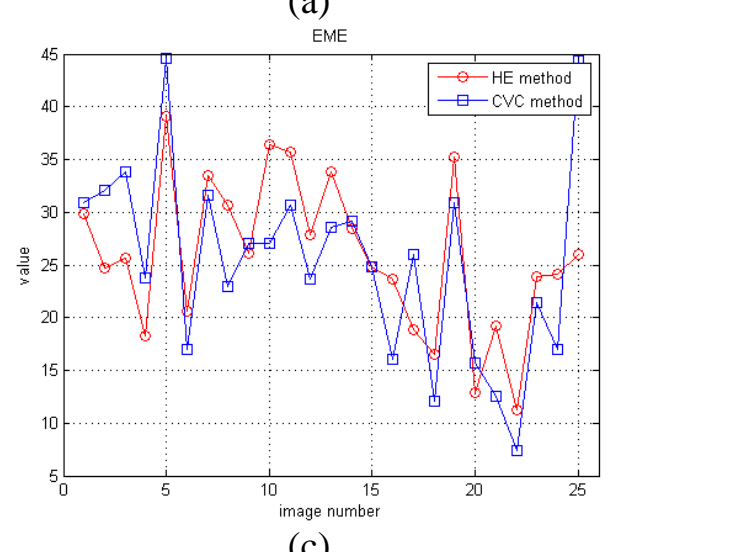

(c)

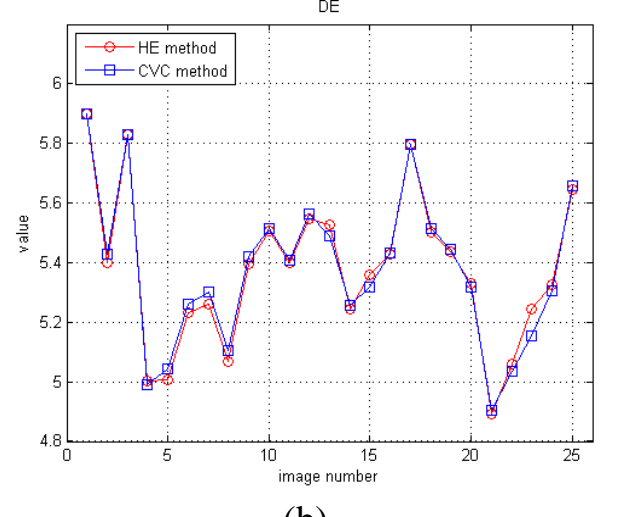

(b)

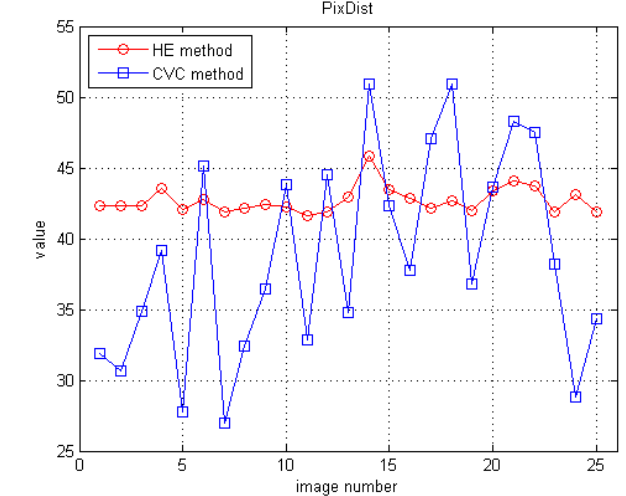

(d)

Figure 17. Objective Performance Results between Two Methods (Quarter Power-consumption): (a) AB, (b) DE, (c) EME, and (d) PixDist

\section{Acknowledgements}

This research was supported by Basic Science Research Program through the National Research Foundation of Korea (NRF) funded by the Ministry of Science, ICT and Future Planning (2013RTA1A1010797)

\section{References}

[1] R. C. Gonzalez and R. E. Woods, "Digital Image Processing”, 3rd ed. Upper Saddle River, NJ: Prentice-Hall, (2006).

[2] R. Dale-Jones and T. Tjahjadi, "A study and modification of the local histogram equalization algorithm," Pattern Recognit., vol. 26, no. 9, (1993) September, pp. 1373-1381.

[3] T. K. Kim, J. K. Paik, and B. S. Kang, "Contrast enhancement system using spatially adaptive histogram equalization with temporal filtering," IEEE Trans. Consum. Electron., vol. 44, no. 1, (1998) February , pp. 82-87.

[4] M. Abdullah-Al-Wadud, M. Kabir, M. Dewan and O. Chae, "A dynamic histogram equalization for image contrast enhancement,” IEEE Trans. Consum. Electron., vol. 53, no. 2, (2007) May, pp. 593-600. 
[5] Q. Wang and R. K. Ward, "Fast image/video contrast enhancement based on weighted thresholded histogram equalization," IEEE Trans. Consum. Electron., vol. 53, no. 2, (2007) May, pp. 757-764.

[6] C.-C. Sun, S.-J. Ruan, M.-C. Shie and T.-W. Pai, "Dynamic contrast enhancement based on histogram specification," IEEE Trans. Consum. Electron., vol. 51, no. 4, (2005) November, pp. 1300-1305.

[7] T. Celik and T. Tjahjadi, "Contextual and variational contrast enhancement," IEEE Trans. Image Process., vol. 20, no. 12, (2011) December, pp. 3431-3441.

[8] S.-D. Chen and A. Ramli, "Minimum mean brightness error bi-histogram equalization in contrast enhancement," IEEE Trans. Consum. Electron., vol. 49, no. 4, (2003) November, pp. 1310-1319.

[9] C. E. Shannon, “A mathematical theory of communication,” Bell Syst.. Tech. J., vol. 27, (1948), pp. 379-423, 623-656.

[10] S. Agaian, B. Silver, and K. Panetta, "Transform coefficient histogram-based image enhancement algorithms using contrast entropy,” IEEE Trans. Image Process., vol. 16, no. 3, (2007) March, pp. 741-758.

\section{Author}

Gwanggil Jeon received the BS, MS, and Ph.D, (sumna cun laude) degrees in Department of Electronics and Computer Engineering from Hanyang University, Seoul, Korea, in 2003, 2005, and 2008, respectively.

From 2008 to 2009, he was with the Department of Electronics and Computer Engineering, Hanyang University, from 2009 to 2011, he was with the School of Information Technology and Engineering (SITE), University of Ottawa, as a postdøetoral fellow, and from 2011 to 2012, he was with the Graduate School of Science \& Technology, Niigata University, as an assistant professor. He is currently an assistant professor with the Department of Embedded Systems Engineering, Incheon National Umiversity, Incheon, Korea. His research interests fall under the umbrella of image processing, particularly image compression, motion estimation, demosaicking, and image enhancement as well as computational intelligence such as fuzzy and rough sets theories.

He was the recipient of the IEEE Chesten Sall Award in 2007 and the 2008 ETRI Journal Paper Award.
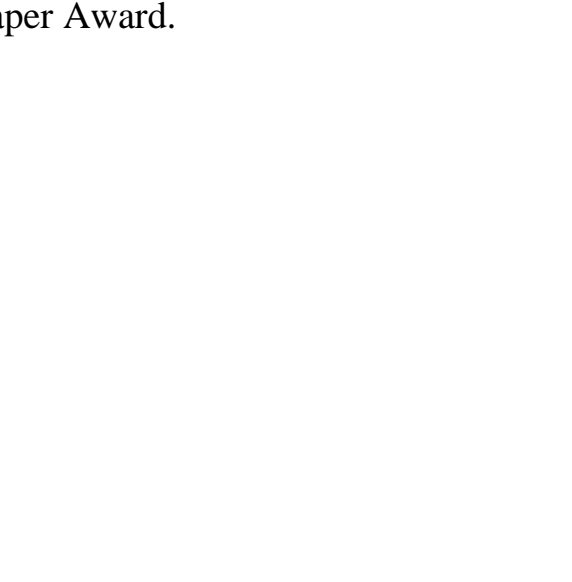